\title{
Identifying a subpopulation with higher likelihoods of early response to treatment in a heterogeneous rare disease: a post hoc study of response to teduglutide for short bowel syndrome
}

This article was published in the following Dove Press journal:

Therapeutics and Clinical Risk Management

\author{
Kristina S Chen' \\ Jipan $\mathrm{Xie}^{2}$ \\ Wenxi Tang ${ }^{3}$ \\ Jing Zhao ${ }^{4}$ \\ Palle B Jeppesen ${ }^{5}$ \\ James E Signorovitch ${ }^{4}$ \\ 'Outcomes Research and \\ Epidemiology, Shire Human Genetic \\ Therapies, Inc., Cambridge, MA, USA; \\ ${ }^{2}$ Analysis Group, Inc., Los Angeles, \\ CA, USA; ${ }^{3}$ Analysis Group, Inc., New \\ York, NY, USA; ${ }^{4}$ Analysis Group, \\ Inc., Boston, MA, USA; ${ }^{5}$ Department \\ of Medical Gastroenterology, \\ Rigshospitalet, Copenhagen, Denmark
}

Correspondence: Kristina S Chen

Shire Human Genetic Therapies, Inc., 650

E Kendall Street, Boston, MA 02I42, USA

Tel + I 617588 874|

Email krchen@shire.com
Purpose: Teduglutide, a glucagon-like peptide-2 analog, has demonstrated efficacy in reducing parenteral support (PS) among patients with short bowel syndrome with intestinal failure (SBS-IF). This study aims to identify a subpopulation of SBS-IF patients for whom teduglutide has an especially pronounced effect.

Patients and methods: Data were from a 24-week, Phase III trial (Study of Teduglutide Effectiveness in Parenteral Nutrition-Dependent SBS Subjects; NCT00798967) that randomized SBS-IF patients with PS dependency to receive teduglutide $(n=43)$ or placebo $(n=43)$. Two prediction models ( 1 for each arm) were developed for response, defined as $20 \%$ reduction in weekly PS at Weeks 20 and 24. Potential predictors included demographics, disease characteristics, and concomitant medications. Patients were then ranked based on the effect score, an individualized predicted response rate difference with teduglutide versus placebo. A subpopulation of patients with a pronounced benefit from teduglutide versus placebo was identified. Baseline characteristics and clinical outcomes were compared between patients included versus those not included in the subpopulation.

Results: Six predictors of response to teduglutide were selected: older age, volvulus as the cause of major intestinal resection, baseline PS volume $>6 \mathrm{~L}$ per week, longer time since start of PS dependency, absence of ileocecal valve, and lower percentage of colon remaining. Higher percentage of colon remaining and volvulus were the selected predictors for response to placebo. A subpopulation of patients more likely to respond to teduglutide was identified as those with the top $60 \%$ effect scores. The difference in response rate between teduglutide and placebo was $62 \%$ in the subpopulation, which was substantially higher than the difference of $33 \%$ in the overall population. Mean PS day reduction was also significantly higher for teduglutide compared to placebo in the subpopulation.

Conclusion: Pretreatment characteristics as predictors of response to teduglutide versus placebo within 24 weeks were identifiable in the clinical trial population of SBS-IF patients.

Keywords: SBS, intestinal failure, teduglutide, subpopulation

\section{Introduction}

Short bowel syndrome (SBS) is a rare malabsorption disorder caused by massive resection of small intestine, congenital defect, or disease-associated loss of absorption. ${ }^{1}$ It is a subcategory of intestinal failure (IF), which is defined as, "the reduction of gut function below the minimum necessary for the absorption of macronutrients and/or water and electrolytes, such that intravenous supplementation is required to maintain 
health and/or growth" according to the 2016 European Society for Clinical Nutrition and Metabolism guidelines. ${ }^{2,3}$ The most common causes of SBS associated with IF (SBS-IF) in adults include Crohn's disease, acute bowel infarction, radiation strictures, and adhesive obstruction. ${ }^{4,5}$ The severity of SBS-IF is affected by factors such as the sites and extent of resection, the quality of the remnant intestinal mucosa, the ability of the patient to compensate orally for malabsorption, and, ultimately, the remnant absorptive capacity of remaining bowels. ${ }^{6-8}$ Although the prevalence of SBS-IF in adults is not known, it has been estimated that $\sim 10,000-20,000$ individuals in the USA and 4 individuals per million in the European population have SBS-IF. ${ }^{9-11}$

A variety of metabolic and physiologic complications are known to be associated with SBS-IF, such as nutrient deficiencies, fluid and electrolyte disturbances, and diarrhea. ${ }^{12}$ Because of functional losses of the resected small intestine, a substantial number of SBS patients require parenteral support (PS) for supplemental nutrients, fluids, and electrolytes, which is potentially associated with severe complications such as liver and kidney damage. ${ }^{10}$ Therefore, treatments improving the intestinal absorption of fluids and nutrients and reducing the frequency and volume of PS are emerging for patients with SBS-IF.

Somatropin, a human growth hormone, and glutamine, an $\alpha$-amino acid, were approved by the US Food and Drug Administration (FDA) to treat SBS patients receiving nutritional support in 2003 and 2004, respectively. ${ }^{13,14}$ Despite the potential positive effect of human growth hormone with or without glutamine on energy absorption and weight gain, the benefit was short lived and did not continue after cessation of the treatment. ${ }^{15}$ Side effects were prominent. In 2012, teduglutide, an analog of glucagon-like peptide-2, was approved by the FDA and European Medicines Agency (EMA) for the treatment of adult patients with SBS-IF receiving nutritional support. In 2016, the EMA approved teduglutide for the treatment of pediatric patients ( $\geq 1$ year) with SBS-IF. It was the third approved treatment by the FDA and the first by the EMA for this indication. ${ }^{16,17}$ Teduglutide has been shown to be efficacious in reducing the need for PS in 24 weeks and during 2 years of follow-up in clinical studies. ${ }^{18,19}$ Although almost all SBS-IF patients with PS dependency eventually responded to teduglutide in the clinical trial, the heterogeneity of treatment response across patient subpopulations was not evaluated. Identification of subpopulations with response to teduglutide can inform the optimization of treatment outcomes and health care resource use. This post hoc study used pretreatment characteristics of the cohort that participated in the teduglutide registration trial to identify a subpopulation of SBS-IF patients for whom teduglutide has an especially pronounced effect during the 24 weeks of follow-up.

\section{Materials and methods Study population}

The present study was a post hoc analysis of the STEPS (Study of Teduglutide Effectiveness in Parenteral NutritionDependent SBS Subjects) trial (NCT00798967), a randomized, double-blind, placebo-controlled Phase III study conducted in 10 countries across Europe and North America. ${ }^{18}$ The trial included adults with a history of SBS-IF that resulted in PS dependency. A total of 86 patients were randomized in a 1:1 ratio to receive placebo or teduglutide $0.05 \mathrm{mg} / \mathrm{kg} /$ day for 24 weeks. The primary endpoint of the STEPS trial was defined as a $20 \%-100 \%$ reduction in weekly PS (ie, parenteral nutrition and/or intravenous fluid volume) from baseline to Week 20, maintained at Week 24. The intentto-treat population from the STEPS trial was included in the current analysis. The trial was approved by local institutional review boards or medical ethics committees (Table S1). All participants provided written informed consent.

\section{Study measures}

Baseline characteristics

Baseline characteristics including patient demographics (ie, age, sex), SBS characteristics (ie, cause of major intestinal resection, presence of stoma, percentage of colon remaining, presence of distal and terminal ileum, presence of ileocecal valve), PS characteristics (ie, baseline PS volume at randomization, time since start of PS dependency), and concomitant narcotics use were collected for the analysis. Missing data in percentage of colon remaining were imputed based on whether a patient had colon in continuity. For patients without colon in continuity, missing data in percentage of colon remaining were imputed as 0 ; for patients with colon in continuity, missing data were imputed as the mean percentage of colon remaining.

\section{Outcomes}

This analysis identified subpopulations of patients that are more likely to achieve response when treated with teduglutide compared to placebo in the 24-week trial. Response was defined as the achievement of $\geq 20 \%$ reduction from baseline in weekly PS at Week 20, maintained at Week 24. Additional outcomes evaluated in the study included reduction in PS days and change in SBS quality of life (QoL) score. PS volume was recorded daily by patients in an electronic diary, 
and reduction in PS days was calculated as the difference in number of days with PS between Week 24 and baseline. ${ }^{18}$ QoL in patients with SBS was evaluated using an SBS-specific patient-reported outcomes QoL instrument (ie, SBS-QoL). ${ }^{20}$ Change in SBS-QoL score was calculated as the difference between Week 24 and baseline. The SBS-QoL score ranged from 0 to 170, representing "perfect" to "worst" QoL, and the minimal clinically important difference was defined as a positive change of score from baseline above the 2-fold measurement error of the SBS-QoL (ie, 18.4). ${ }^{21}$

\section{Analysis \\ Descriptions of baseline characteristics and outcomes in the overall population}

Baseline characteristics and outcomes were summarized for the teduglutide and placebo arms. Means and SDs were reported for continuous variables and counts, and proportions were reported for categorical variables. Comparisons between teduglutide and placebo arms were conducted using Wilcoxon rank-sum tests for continuous characteristics and chi-square or Fisher's exact tests (if expected cell counts $<5$ ) for categorical characteristics.

\section{Prediction of responses to teduglutide and placebo}

To identify patients with an especially pronounced 24 -week treatment benefit from teduglutide versus placebo, we first ranked them based on predicted level of benefit from teduglutide. The predicted level of benefit for each patient was estimated as the difference between his or her predicted response rates with teduglutide or placebo. These individualized predictions of response rates were obtained from multivariable models fit to each arm. For the teduglutide arm, a prediction model for response was estimated using logistic regression. Ten baseline characteristics were considered, based on clinical input and data availability, as candidate predictors, including age, sex, cause of major intestinal resection, presence of stoma, percentage of colon remaining, presence of distal/terminal ileum, presence of ileocecal valve, baseline PS volume at randomization, and time since start of PS dependency. To identify a parsimonious model, and to account for the limited sample size and avoid overfitting, a penalized regression approach was used to fit the model and to select a subset of important predictors from the list of candidates. ${ }^{22,23}$ The same approach was used to develop a separate prediction model for the placebo arm. The in-sample predictive performance and calibration of each prediction model was evaluated using $\mathrm{c}$ statistics and Hosmer-Lemeshow tests. ${ }^{24}$

\section{Identification of a higher response subpopulation for} teduglutide

After developing the aforementioned prediction models, an effect score, an individualized predicted response rate difference with teduglutide versus placebo based on the prediction models, was estimated for individual patients. Patients were then ranked according to this effect score. This ranking provides a basis for defining subpopulations that exhibit treatment effect heterogeneity. Highly ranked patients will show greater benefits for teduglutide in 24 weeks; lowerranked patients will show smaller benefits at that time point. Subpopulations can be defined by applying thresholds to the effect score (Figure S1). This approach has been used to identify subpopulations that benefit most from treatment in previous clinical trials. ${ }^{22,25-28}$

A systematic approach to evaluating these subpopulations borrows an idea from economics: the efficiency frontier or selection impact curve. ${ }^{29}$ For any subpopulation, we can ask 2 questions: 1) what fraction of the total population falls into the subpopulation and 2) what response rate would we expect in the full population if teduglutide was used only in the subpopulation. At one extreme, the effect score threshold can be set to the highest observed value. The fraction of the population exceeding this threshold is $0 \%$, and the estimated response rate in the full population with $0 \%$ receiving teduglutide is equivalent to the response rate in the placebo arm of the STEPS trial. At the other extreme, the effect score threshold is set below the lowest observed value. The fraction of the population exceeding this threshold is $100 \%$, and the estimated response rate in the full population with $100 \%$ receiving teduglutide is equivalent to the response rate in the teduglutide arm of STEPS. Between these 2 extremes, a series of increasing effect score thresholds will describe a relationship between treating more patients with teduglutide, starting with those expected to benefit most and incrementally expanding to those expected to benefit least, and the overall response rate in the full population. The curve linking these scenarios and describing the relationship between the proportions of patients treated and population response rate is an efficiency frontier, which represents the highest response rate that can be achieved for each level of teduglutide use based on the effect scores.

In the present study, patients were ranked by their individual effect scores from highest to lowest and were sequentially grouped as potential subpopulations by $10 \%$ increments, starting from the top $20 \%$ of patients with the highest individual effect score until all patients (100\%) were included. The initial threshold of $20 \%$ was selected to ensure 
that at least 10 patients were included in the subpopulation. Within each of these subpopulations, the observed response rates to teduglutide and placebo and the difference in these observed response rates were estimated. Response rate in the overall population was estimated using a hypothetical assignment rule where patients in the potential subpopulations received teduglutide and the rest of the population received placebo. The efficiency frontier was then plotted as the convex hull of the size of the potential subpopulations (horizontal axis) and the corresponding response rates in the overall population (vertical axis). Bootstrapping was applied to estimate the $95 \% \mathrm{CI}$ for the efficiency frontier. Based on the efficiency frontier, a subpopulation of patients with a particularly pronounced response rate benefit from teduglutide versus placebo was identified. To characterize this subpopulation, baseline characteristics were summarized and compared between patients included versus not included in the subpopulation. Clinical outcomes, including response rate, reduction in PS days, and change in SBS-QoL, were also summarized and compared. Descriptive summaries were conducted using SAS 9.4 (SAS Institute, Inc., Cary, NC, USA). All other analyses were performed using $\mathrm{R}$ version 3.3.2 (http://cran.r-project.org).

\section{Results \\ Baseline characteristics and prespecified trial analysis outcomes}

All patients in the trial $(\mathrm{N}=86)$ were included in the analysis; 43 were randomized to teduglutide and 43 were randomized to placebo. Baseline characteristics were generally well balanced between the teduglutide and placebo arms (Table 1), with the exception of presence of ileocecal valve, which was significantly less frequent in the teduglutide arm compared to the placebo arm $(7.0 \%$ versus $23.3 \%, P=0.04)$. Mean age was comparable in the teduglutide (50.9 years) and placebo (49.7 years) arms, and the majority of the patients were white (teduglutide: 97.7\%; placebo: 95.3\%; $P$-value not significant). Proportions of men were $48.8 \%$ and $44.2 \%$ in the teduglutide and placebo arms, respectively. Mesenteric vascular disease was the most common cause of major intestinal resection in this patient population; $83 \%$ of patients had a PS volume $>6 \mathrm{~L} /$ week at randomization, with a mean duration of PS dependency of 6.4 years.

Overall, the response rate was significantly higher in patients treated with teduglutide compared to placebo based on data collected at Weeks 20 and 24 (teduglutide versus placebo: $62.8 \%$ versus $30.2 \%, P<0.01$ ). The aforementioned

Table I Baseline characteristics of SBS-IF patients in the STEPS trial

\begin{tabular}{|c|c|c|c|}
\hline Characteristics & $\begin{array}{l}\text { Teduglutide } \\
(n=43)\end{array}$ & $\begin{array}{l}\text { Placebo } \\
(n=43)\end{array}$ & $P$-value \\
\hline \multicolumn{4}{|l|}{ Demographics } \\
\hline Age (years), mean (SD) & $50.9(12.6)$ & $49.7(15.6)$ & 0.82 \\
\hline Male, $\mathrm{n}(\%)$ & $21(48.8)$ & $19(44.2)$ & 0.67 \\
\hline White, n (\%) & $42(97.7)$ & $4 \mathrm{I}(95.3)$ & 1.00 \\
\hline \multicolumn{4}{|l|}{ Baseline SBS characteristics } \\
\hline \multicolumn{4}{|l|}{ Cause of major intestinal resection, $\mathrm{n}(\%)$} \\
\hline Crohn's disease & $10(23.3)$ & $8(18.6)$ & 0.60 \\
\hline Vascular disease & $13(30.2)$ & $16(37.2)$ & 0.49 \\
\hline Injury & $4(9.3)$ & $4(9.3)$ & 1.00 \\
\hline Volvulus & $3(7.0)$ & $6(14.0)$ & 0.48 \\
\hline Cancer & I (2.3) & $2(4.7)$ & 1.00 \\
\hline Other & $12(27.9)$ & $7(16.3)$ & 0.19 \\
\hline Presence of stoma, n (\%) & $21(48.8)$ & $17(39.5)$ & 0.39 \\
\hline Percentage of colon remaining, mean (SD) & $33.9(31.8)$ & $40.9(40.6)$ & 0.39 \\
\hline Presence of distal/terminal ileum, $n(\%)$ & $10(23.3)$ & $14(32.6)$ & 0.34 \\
\hline Presence of ileocecal valve, $n(\%)$ & $3(7.0)$ & $10(23.3)$ & $0.04 *$ \\
\hline \multicolumn{4}{|l|}{ Baseline PS characteristics } \\
\hline Baseline PS volume at randomization, $n$ (\%) & & & 0.78 \\
\hline$\leq 6 \mathrm{~L} /$ week & $8(18.6)$ & $7(16.3)$ & \\
\hline$>6 \mathrm{~L} /$ week & $35(81.4)$ & $36(83.7)$ & \\
\hline Time since start of PS dependency (years), mean (SD) & $6.8(6.3)$ & $5.9(5.7)$ & 0.76 \\
\hline Concomitant narcotics use, $\mathrm{n}(\%)$ & $15(34.9)$ & $12(27.9)$ & 0.49 \\
\hline
\end{tabular}

Note: $* p<0.05$.

Abbreviations: PS, parenteral support; SBS, short bowel syndrome; SBS-IF, SBS with intestinal failure; STEPS, Study of Teduglutide Effectiveness in Parenteral NutritionDependent SBS Subjects. 
results were obtained from the prespecified analysis of the STEPS trial. ${ }^{18}$

\section{Prediction models for response}

A total of 6 baseline characteristics, including age, cause of major intestinal resection, baseline PS volume, time since start of PS dependency, presence of ileocecal valve, and percentage of colon remaining, were selected as predictors for response to teduglutide (Table 2). Among the selected predictors for response to teduglutide, older age (odds ratio 1.023), volvulus as the cause of major intestinal resection (odds ratio 3.765), baseline PS volume $>6 \mathrm{~L}$ per week (odds ratio 4.750), and longer time since start of PS dependency (odds ratio 1.010) were associated with higher odds of response to teduglutide; presence of ileocecal valve (odds ratio 0.550) and higher percentage of colon remaining (odds ratio 0.980 ) were associated with lower odds of response to teduglutide. Two characteristics, percentage of colon remaining and cause of major intestinal resection, were selected as predictors for response to placebo (Table 2). Specifically, higher percentage of colon remaining (odds ratio 1.003) and volvulus as the cause of major intestinal resection (odds ratio 1.238) were associated with higher odds of response to placebo. $\mathrm{C}$ statistics suggested good performance in both prediction models (teduglutide: 0.88 ; placebo: 0.74 ). The models were well calibrated (Hosmer-Lemeshow test $P$-values of 0.15 and 0.26 for teduglutide and placebo, respectively).

\section{A higher response subpopulation for teduglutide}

Differences in response to teduglutide and placebo across the studied range of subpopulations are depicted in Figure 1.
In the subpopulation of patients with the highest $20 \%$ of effect scores (ie, effect score $\geq 0.54$ ), patients treated with teduglutide demonstrated a higher response rate compared to those treated with placebo by $+80.0 \%$. The difference in response rates between teduglutide and placebo decreased when more patients with lower effect scores were included in the subpopulation. When all patients were included (ie, 100\% of the study sample), the difference in response rates between teduglutide and placebo was $+32.6 \%$, the same rate reported in the clinical trial. ${ }^{18}$ This treatment effect heterogeneity was attributable to both increased response rates to teduglutide and decreased response rates to placebo as the effect score increased (Figure 2).

The efficiency frontier, shown in Figure 3, describes the tradeoffs between the size of the subpopulation receiving teduglutide and the resulting response rate in the overall population. The observed probability of response in the overall population increased when more patients received teduglutide. However, the curve reaches a shoulder as the percentage of patients receiving teduglutide exceeds $60 \%$, indicating that most of the teduglutide treatment benefit for overall response rate was achieved when $\sim 60 \%$ of the patients received teduglutide. Therefore, a subpopulation of patients more likely to respond to teduglutide was identified as the patients with the top $60 \%$ effect scores.

Patients in this higher-response subpopulation $(n=51)$ had a mean age of 52.0 years, balanced sex (51.0\% male), and Crohn's disease as the most common cause of major intestinal resection $(31.4 \%)$. The majority of these patients were without distal/terminal ileum or ileocecal valve (Table 3 ). Compared to the lower-response subpopulation $(n=35)$, proportion with colon in continuity $(35.3 \%$ versus $88.6 \%$,

Table 2 Prediction models for response to teduglutide and placebo

\begin{tabular}{|c|c|c|c|c|}
\hline \multirow[t]{2}{*}{ Predictors selected by regression models } & \multicolumn{2}{|l|}{ Teduglutide } & \multicolumn{2}{|l|}{ Placebo } \\
\hline & Coefficient & $\begin{array}{l}\text { Odds } \\
\text { ratio }\end{array}$ & Coefficient & $\begin{array}{l}\text { Odds } \\
\text { ratio }\end{array}$ \\
\hline Intercept & -1.263 & & -0.988 & \\
\hline Percentage of colon remaining & -0.020 & 0.980 & 0.003 & 1.003 \\
\hline Cause of major intestinal resection (volvulus versus other) & 1.326 & 3.765 & 0.214 & 1.238 \\
\hline Presence of ileocecal valve (yes versus no) & -0.598 & 0.550 & & \\
\hline Age (years) & 0.023 & 1.023 & & \\
\hline Baseline PS volume at randomization ( $>6 \mathrm{~L}$ per week versus $\leq 6 \mathrm{~L}$ per week) & 1.558 & 4.750 & & \\
\hline Time since start of PS dependency (years) & 0.010 & 1.010 & & \\
\hline \multicolumn{5}{|l|}{ Model performance } \\
\hline C statistics ${ }^{\mathrm{a}}$ & 0.88 & & 0.74 & \\
\hline$P$-value of Hosmer-Lemeshow test ${ }^{b}$ & 0.15 & & 0.26 & \\
\hline
\end{tabular}

Notes: ${ }^{\mathrm{A}} \mathrm{A} C$ statistic of I corresponds to perfect prediction; a $\mathrm{C}$ statistic of 0.5 corresponds to a prediction rule that does not perform any better than a random prediction. ${ }^{b}$ The Hosmer-Lemeshow test is a goodness-of-fit test. $P<0.05$ indicates a lack of fit.

Abbreviation: PS, parenteral support. 


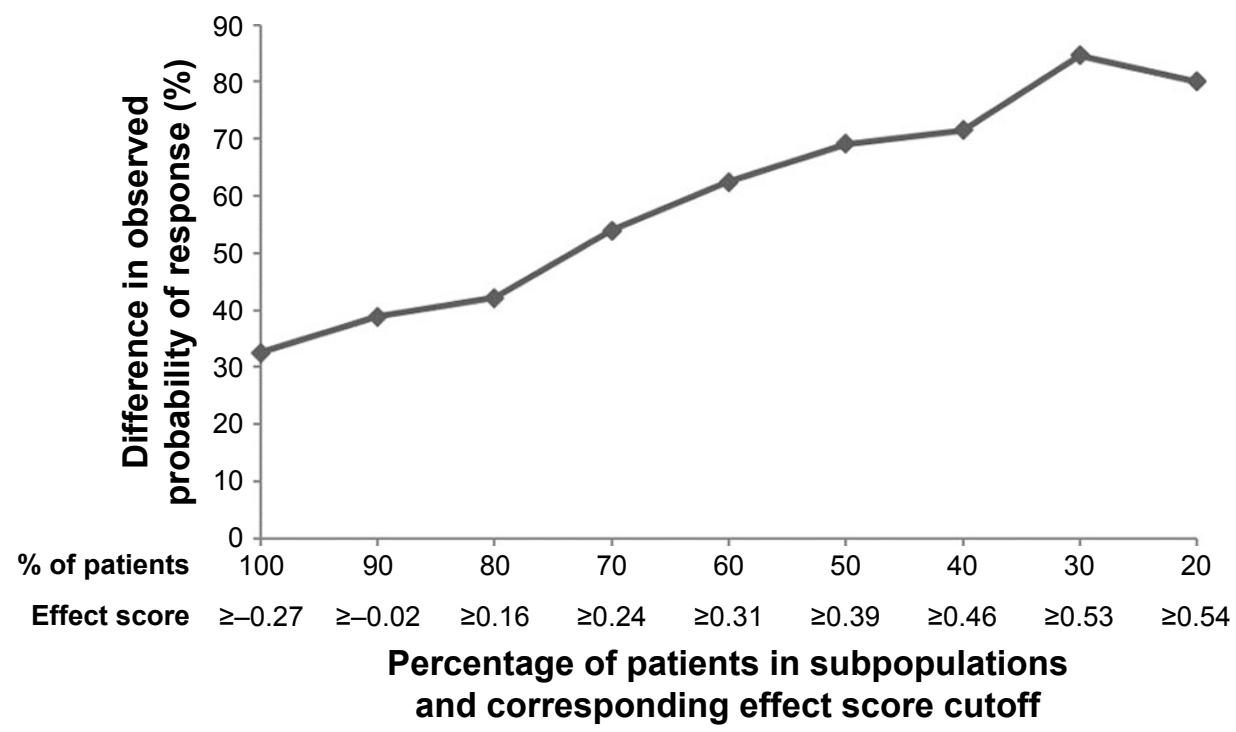

Figure I Difference in response between teduglutide and placebo in potential subpopulations.

$P<0.01)$ and percentage of colon remaining $(18.5$ versus 65.0, $P<0.01)$ were significantly lower in the higherresponse subpopulation.

Clinical outcomes were also evaluated within and between subpopulations (Table 4). Among patients in the higher-response subpopulation, patients treated with teduglutide had significantly better response rate $(87.5 \%$ versus $25.9 \%, P<0.01)$ and significantly higher mean reduction in PS days ( 1.0 versus $0.4, P=0.01$ ), compared to those treated with placebo. The difference in response rate between teduglutide and placebo was $61.6 \%$ in the higher response subpopulation, which was also substantially higher than the difference of $32.6 \%$ in the overall population. In contrast, all evaluated clinical outcomes (ie, response, reduction in PS days, and change in SBS-QoL) were comparable between teduglutide- and placebo-treated patients among the lowerresponse subpopulation (ie, patients with lower $60 \%$ effect scores; Table 4).

\section{Discussion}

The present study evaluated the predictability of response to teduglutide and to use a model-based individual effect score to identify patients who are more likely to achieve a response to teduglutide in patients with SBS-IF at 24 weeks. Within the Phase III STEPS trial, we were able to accurately predict response among patients treated with teduglutide

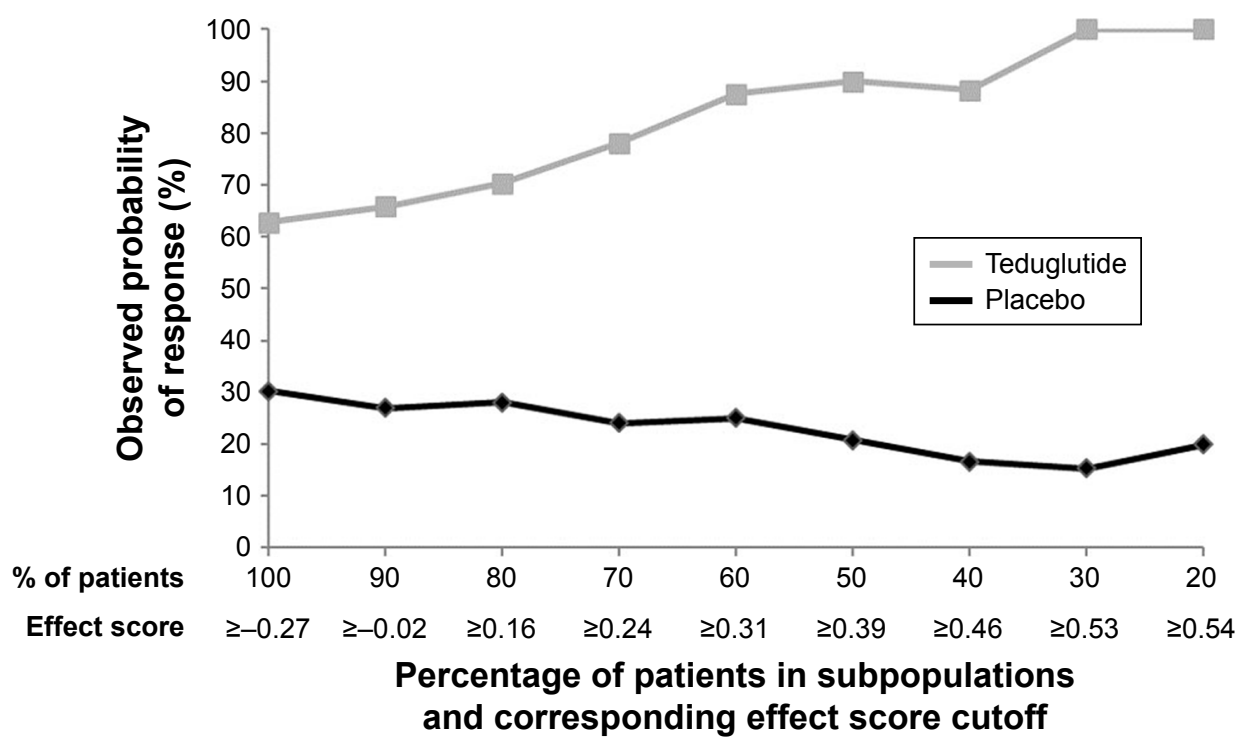

Figure 2 Response in potential subpopulations by treatment. 


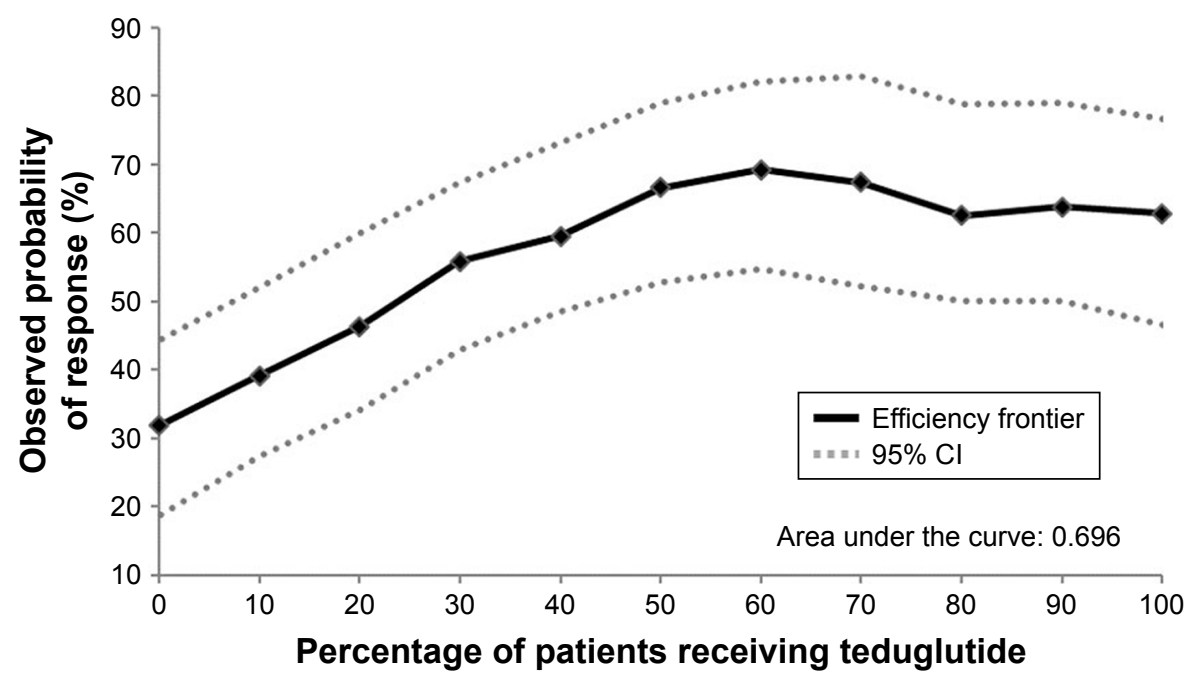

Figure 3 Efficiency frontier.

and placebo based on patient characteristics measured at baseline. These predictive models were used to identify a subpopulation of patients who were especially likely to experience a response to teduglutide within 24 weeks. Within this subpopulation, the increase in the response rate with teduglutide versus placebo exceeded $60 \%$, nearly double the effect size in the overall population. The subpopulation was identified without cherry-picking or data-dredging, and the

Table 3 Comparison of baseline characteristics in the higher- and lower-response subpopulations

\begin{tabular}{|c|c|c|c|}
\hline Baseline characteristics & $\begin{array}{l}\text { Higher-response } \\
\text { subpopulation } \\
(n=5 \text { I) }\end{array}$ & $\begin{array}{l}\text { Lower-response } \\
\text { subpopulation } \\
(n=35)\end{array}$ & P-value \\
\hline \multicolumn{4}{|l|}{ Demographics } \\
\hline Age (years), mean (SD) & $52.0(14.1)$ & $47.8(14.0)$ & 0.27 \\
\hline Male, n (\%) & $26(5 I .0)$ & $14(40.0)$ & 0.32 \\
\hline White, n (\%) & $48(94.1)$ & $35(100.0)$ & 0.27 \\
\hline \multicolumn{4}{|l|}{ Disease characteristics } \\
\hline \multicolumn{4}{|l|}{ Cause of major intestinal resection, $\mathrm{n}(\%)$} \\
\hline Crohn's disease & $16(31.4)$ & $2(5.7)$ & $<0.0 I^{*}$ \\
\hline Vascular disease & $10(19.6)$ & $19(54.3)$ & $<0.01 *$ \\
\hline Injury & $4(7.8)$ & $4(\mathrm{I} \mid .4)$ & 0.71 \\
\hline Volvulus & $7(13.7)$ & $2(5.7)$ & 0.30 \\
\hline Cancer & $3(5.9)$ & $0(0.0)$ & 0.27 \\
\hline Other & $\mathrm{II}(2 \mathrm{I} .6)$ & $8(22.9)$ & 0.89 \\
\hline Colon in continuity, $\mathrm{n}(\%)$ & $18(35.3)$ & $31(88.6)$ & $<0.01 *$ \\
\hline Percentage of colon remaining, mean (SD) & $18.5(26.3)$ & $65.0(31.3)$ & $<0.0 I^{*}$ \\
\hline Estimated remaining small intestine length $(\mathrm{cm})$, mean $(\mathrm{SD})$ & $87.0(70.6)$ & $61.6(51.7)$ & 0.07 \\
\hline Estimated remaining small intestine length $(\mathrm{cm}), \mathrm{n}(\%)$ & & & 0.11 \\
\hline$<60$ & $20(42.6)$ & $20(60.6)$ & \\
\hline$\geq 60$ & $27(57.4)$ & $13(39.4)$ & \\
\hline Presence of distal/terminal ileum, n (\%) & $9(17.6)$ & $15(42.9)$ & $0.01^{*}$ \\
\hline Presence of ileocecal valve, n (\%) & $2(3.9)$ & II (3I.4) & $<0.01 *$ \\
\hline Presence of stoma, n (\%) & $33(64.7)$ & $5(14.3)$ & $<0.0 I^{*}$ \\
\hline Time since last small bowel resection (years), mean (SD) & $6.6(6.1)$ & $8.2(8.6)$ & 0.88 \\
\hline \multicolumn{4}{|l|}{ Baseline PS characteristics } \\
\hline Baseline PS volume at randomization, n (\%) & & & $<0.01 *$ \\
\hline$\leq 6 \mathrm{~L} /$ week & I $(2.0)$ & $14(40.0)$ & \\
\hline$>6 \mathrm{~L} /$ week & $50(98.0)$ & $21(60.0)$ & \\
\hline Time since start of PS dependency (years), mean (SD) & $6.7(5.8)$ & $5.8(6.3)$ & 0.20 \\
\hline Concomitant narcotics use, n (\%) & $16(31.4)$ & II (3I.4) & 1.00 \\
\hline
\end{tabular}

Note: ${ }^{*} P<0.05$

Abbreviation: PS, parenteral support. 
Table 4 Comparison of clinical outcomes between teduglutide and placebo in subpopulations

\begin{tabular}{|c|c|c|c|c|c|c|}
\hline \multirow[t]{2}{*}{ Clinical outcome } & \multicolumn{3}{|c|}{ Higher-response subpopulation } & \multicolumn{3}{|c|}{ Lower-response subpopulation } \\
\hline & $\begin{array}{l}\text { Teduglutide } \\
(n=24)\end{array}$ & $\begin{array}{l}\text { Placebo } \\
(n=27)\end{array}$ & $P$-value & $\begin{array}{l}\text { Teduglutide } \\
(n=19)\end{array}$ & $\begin{array}{l}\text { Placebo } \\
(n=16)\end{array}$ & $P$-value \\
\hline Response rate, $\mathrm{n}(\%)$ & $21(87.5)$ & $7(25.9)$ & $<0.0 I^{*}$ & $6(31.6)$ & $6(37.5)$ & 0.71 \\
\hline Reduction in PS days, mean (SD) & $1.0(1.2)$ & $0.4(0.9)$ & $0.01^{*}$ & $0.8(1.0)$ & $0.7(0.9)$ & 0.51 \\
\hline Change in SBS-QoL, mean (SD) & $-12.2(31.3)$ & $-8.5(31.1)$ & 0.57 & $-11.2(20.5)$ & $-2.6(30.2)$ & 0.59 \\
\hline
\end{tabular}

Note: ${ }^{*} p<0.05$

Abbreviations: PS, parenteral support; QoL, quality of life; SBS, short bowel syndrome.

key subjective decision was the list of candidate predictors, which was specified before data analysis. It is worth noting that the present study investigated predictors of outcomes among SBS patients who were PS dependent for at least 12 months following intestinal resection. The identified predictors may, therefore, differ from those for postsurgical outcomes among recently resected patients.

Diversity in response to treatment may occur because of patients' individual disease characteristics, variations in genetic composition, and environmental factors. ${ }^{30,31}$ Specifically, individualized management for patients with SBS-IF should be considered given variations in diagnosis, remaining bowel length/function, and psychosocial characteristics. ${ }^{32}$ Optimal treatment strategies can be informed by an understanding of how individual patients may respond differently to treatment. The UK National Institute for Health and Care Excellence has published a review on how subpopulations should be considered to maximize health gains for both clinical efficacy and cost-effectiveness. ${ }^{33}$ Guidelines like this have provided foundations for the development of algorithms and methods for subpopulation identification.

In rare diseases, there is often more heterogeneity among patients, which further complicates the understanding of treatment benefit. It is important to consider different patient characteristics in support of individualized treatment approaches. The US National Institutes of Health has developed a program called Therapeutics for Rare and Neglected Diseases, which may help to optimize the treatment effects on rare diseases by identifying subgroups of patients who can benefit more from a potential drug. ${ }^{30}$ Using clinical trial data, the current study demonstrates substantial heterogeneity in response to treatment among patients with SBS-IF. Using appropriate methods, we are able to identify a higher-response subpopulation even with a limited sample size. Results from this study may help guide more efficient treatment and management of SBS-IF. The approach could also potentially be applied to other rare disease areas to identify the right population for the right treatment.
The present study has shown that identifying patient and disease characteristics associated with response to teduglutide within 24 weeks is feasible among SBS-IF patients. Patients in the identified subpopulation were characterized by absence of distal/terminal ileum or ileocecal valve, a lower likelihood of having colon in continuity, and a lower percentage of colon remaining. These patient characteristics are well supported by the phenotypic feature of SBS-IF patients. Specifically, SBS-IF patients with a preserved terminal ileum and ileocecal valve and a substantial part of the colon are characterized by having more normal gastric emptying, gastric acid secretion, and a good structural and functional adaptation compared to patients with a jejunostomy. This may not only relate to the fluid, electrolyte, and energy-salvaging effects of the preserved colon, but also to a preserved or even elevated endogenous hormone secretion and neuroendocrine feedback signaling from the distal to the proximal gastrointestinal tract. Therefore, the effect of and response to exogenous supplementation of the glucagon-like peptide-2 (GLP-2) analog teduglutide may be better in the patients with a jejunostomy who lack their endogenous postprandial GLP-2 secretion and are characterized phenotypically by accelerated gastric emptying, gastric hypersecretion, and poor adaptation following resection.

Based on the clinical efficacy reported in the STEPS-2 trial (ie, the extension study of the STEPS trial), the majority of the patients that did not respond to teduglutide within 24 weeks eventually responded during the extension phase. Therefore, this analysis does not lead to the identification of a specific etiology for response to teduglutide or patient selection criteria for teduglutide treatment among SBS-IF patients but aims instead at identifying characteristics linked to an earlier response to teduglutide within 24 weeks. Because the large majority of patients with SBS-IF eventually respond to teduglutide treatment, knowing whether they are likely to achieve an earlier response within 24 weeks may help set more accurate patient and physician expectations and encourage treatment persistence. For patients with characteristics of 
the lower-response subpopulation, knowing that a longer time to response is typical for their patient profile could prevent early treatment discontinuation and support waiting for longterm treatment benefits. Thus, the clinical implications of this research are more focused on setting appropriate expectations and managing adherence to teduglutide than informing which patients should or should not be treated.

This study comes with some limitations. First, the data used in the study were from a clinical trial population comprising only patients that met specific inclusion and exclusion criteria. Therefore, the results may not be generalizable to other SBS-IF populations. Second, the candidate baseline characteristics for predicting response were limited in number because of the small sample size and were selected among the characteristics collected during the baseline period of the clinical trial. There may be important subpopulations defined by as-yet unknown or unmeasured biomarkers or clinical characteristics. Finally, the statistical power of the analysis and the robustness of the prediction models may be limited by small sample size.

In conclusion, teduglutide has demonstrated better response within 24 weeks compared to placebo in a randomized, double-blind, placebo-controlled Phase III clinical trial of an SBS-IF population. The present study was able to identify pretreatment characteristics as predictors of response to teduglutide versus placebo within 24 weeks and a higher-response subpopulation for this time period with a limited study sample. The analytical approach can potentially be applied to other disease areas to inform more efficient treatment and management of the disease. Research in personalized treatment selection will serve as an important step in improving patient care and optimizing treatment efficiency. The current analysis is considered exploratory, and further analysis to prospectively assess reproducibility of the results in a separate population is warranted.

\section{Acknowledgments}

We gratefully acknowledge the contribution of Clément Olivier, MD, of Shire International GmbH (Zug, Switzerland), who provided input in clinical knowledge on the disease, the treatment, and the clinical trial data.

Presented by Chen K, Xie J, Tang C, et al. Identifying higher-value subpopulations for treatment in heterogeneous rare diseases: an example study of early responders to teduglutide for short bowel syndrome. Poster presented at the International Society for Pharmacoeconomics and Outcomes Research Annual European Congress; October 29November 2, 2016; Vienna, Austria.

\section{Author contributions}

All authors contributed toward data analysis, drafting and revising the paper and agree to be accountable for all aspects of the work.

\section{Disclosure}

$\mathrm{KSC}$ is an employee of Shire. PBJ has served as a speaker bureau member and consultant for Shire, and as a study investigator, consultant, and advisory board member for NPS Pharmaceuticals, Inc. JX, JES, WT, and JZ are employees of Analysis Group, Inc., which received payment from Shire for contracted research. The authors report no other conflicts of interest in this work.

\section{References}

1. O'Keefe SJ, Buchman AL, Fishbein TM, Jeejeebhoy KN, Jeppesen PB, Shaffer J. Short bowel syndrome and intestinal failure: consensus definitions and overview. Clin Gastroenterol Hepatol. 2006;4(1):6-10.

2. Pironi L, Arends J, Bozzetti F, et al. ESPEN guidelines on chronic intestinal failure in adults. Clin Nutr. 2016;35(2):247-307.

3. Pironi L. Definitions of intestinal failure and the short bowel syndrome. Best Pract Res Clin Gastroenterol. 2016;30(2):173-185.

4. Sturm A, Layer P, Goebell H, Dignass AU. Short-bowel syndrome: an update on the therapeutic approach. Scand J Gastroenterol. 1997; 32(4):289-296.

5. Thompson JS. Inflammatory disease and outcome of short bowel syndrome. Am J Surg. 2000;180(6):551-554.

6. Lykins TC, Stockwell J. Comprehensive modified diet simplifies nutrition management of adults with short-bowel syndrome. J Am Diet Assoc. 1998;98(3):309-315.

7. Thompson JS. Management of the short bowel syndrome. Gastroenterol Clin North Am. 1994;23(2):403-420.

8. Ukleja A, Tammela LJ, Lankisch MR, Scolapio JS. Nutritional support for the patient with short-bowel syndrome. Curr Gastroenterol Rep. 1999;1(4):331-334.

9. Weireiter L. Nutritional hope or hype for short bowel syndrome? Am J Gastroenterol. 1996;91(10):2246-2247.

10. Sundaram A, Koutkia P, Apovian CM. Nutritional management of short bowel syndrome in adults. J Clin Gastroenterol. 2002;34(3): 207-220.

11. Jeppesen PB. Spectrum of short bowel syndrome in adults: intestinal insufficiency to intestinal failure. JPEN J Parenter Enteral Nutr. 2014; 38(1 Suppl):8S-13S.

12. Escott-Stump S. Nutrition and Diagnosis-Related Care, 3rd Edition Philadelphia, PA, USA: Lea \& Febiger; 1992.

13. Zorbtive ${ }^{\circledR}$ [package insert]. Rockland, MA, USA: EMD Serono, Inc.; 2003.

14. NUTRESTORE ${ }^{\circledR}$ [package insert]. Torrance, CA, USA: Emmaus Medical, Inc.; 2004.

15. Wales PW, Nasr A, de Silva N, Yamada J. Human growth hormone and glutamine for patients with short bowel syndrome. Cochrane Database Syst Rev. 2010;(6):CD006321.

16. GATTEX ${ }^{\circledR}$ [package insert]. Bedminster, NJ, USA: NPS Pharmaceuticals; 2012.

17. Revestive - Summary of Product Characteristics (SPC). [online] Available from: http://www.ema.europa.eu/docs/en_GB/document_library/ EPAR_-_Product_Information/human/002345/WC500132926.pdf. Accessed July 16, 2017.

18. Jeppesen PB, Pertkiewicz M, Messing B, et al. Teduglutide reduces need for parenteral support among patients with short bowel syndrome with intestinal failure. Gastroenterology. 2012;143(6):1473.e3-1481.e3. 
19. Schwartz LK, O’Keefe SJ, Fujioka K, et al. Long-term teduglutide for the treatment of patients with intestinal failure associated with short bowel syndrome. Clin Transl Gastroenterol. 2016;7:e142.

20. Berghofer P, Fragkos KC, Baxter JP, et al. Development and validation of the disease-specific Short Bowel Syndrome-Quality of Life (SBS-QoL ${ }^{\mathrm{TM}}$ ) scale. Clin Nutr. 2013;32(5):789-796.

21. Jeppesen PB, Pertkiewicz M, Forbes A, et al. Quality of life in patients with short bowel syndrome treated with the new glucagon-like peptide-2 analogue teduglutide-analyses from a randomised, placebo-controlled study. Clin Nutr. 2013;32(5):713-721.

22. Cai T, Tian L, Wong PH, Wei LJ. Analysis of randomized comparative clinical trial data for personalized treatment selections. Biostatistics. 2011;12(2):270-282.

23. Tibshirani R. Regression shrinkage and selection via the lasso. J R Stat Soc Series B Methodol. 1996;58(1):267-288.

24. David WH Jr, Stanley L, Rodney XS. Applied Logistic Regression, 3rd Edition. New York, NY, USA: Wiley; 2013.

25. Tian L, Cai T, Wei L. Identifying subjects who benefit from additional information for better prediction of the outcome variables. Biometrics. 2009;65(3):894-902.

26. Zhao L, Tian L, Cai T, Claggett B, Wei LJ. Effectively selecting a target population for a future comparative study. J Am Stat Assoc. 2013; 108(502):527-539.
27. Li J, Zhao L, Tian L, et al. A predictive enrichment procedure to identify potential responders to a new therapy for randomized, comparative controlled clinical studies. Biometrics. 2016;72(3):877-887.

28. Claggett B, Tian L, Castagno D, Wei LJ. Treatment selections using risk-benefit profiles based on data from comparative randomized clinical trials with multiple endpoints. Biostatistics. 2015;16(1):60-72.

29. Song X, Pepe MS. Evaluating markers for selecting a patient's treatment. Biometrics. 2004;60(4):874-883.

30. Hamburg MA, Collins FS. The path to personalized medicine. $N$ Engl J Med. 2010;363(4):301-304.

31. Ginsburg GS, Willard HF. Genomic and personalized medicine: foundations and applications. Transl Res. 2009;154(6):277-287.

32. Nightingale J, Woodward JM; Small Bowel and Nutrition Committee of the British Society of Gastroenterology. Guidelines for management of patients with a short bowel. Gut. 2006;55(Suppl 4):iv1-iv12.

33. Sculpher M. Briefing Paper for Methods Review Workshop on Identifying Sub-groups and Exploring Heterogeneity. London: National Institute for Health and Clinical Excellence; 2007. 


\section{Supplementary materials}

Table SI List of Independent Ethics Committees or Institutional Review Boards that approved the study

\begin{tabular}{ll}
\hline Center number & Ethics Committee or Institutional Review Board \\
\hline 0109 & University of Pennsylvania Institutional Review Board \\
0111 & Copernicus Group Institutional Review Board \\
0115 & Cleveland Clinic Institutional Review Board \\
0144 & Institutional Review Board: Program for the Protection of Human Subjects \\
0201 & Vanderbilt University Medical Center Institutional Review Board \\
0220 & Copernicus Group Institutional Review Board \\
0101 & University Health Network Research Ethics Board \\
0106 & St Michael's Research Ethics Board \\
0202 & Hamilton Health Sciences Corporation Research Ethics Board \\
0218 & Health Research Ethics Board \\
$0138,0210,0219,0155$ & Komisja Bioetyczna przy Warzawskim Uniwersytecie Medyczynym \\
0135,0209 & CPP lle de France VIII \\
0147 & Ethik-Kommission des Landes Berlin \\
0214 & Landesamt für Gesundheit und Soziales Berlin Ethik-Kommission des Landes Berlin \\
0203 & Comitato Etico dell' Azinda \\
0207 & Comitato Etico dell' Università degli Studi di Napoli Federico II \\
0211 & Comitato Etico Dell' Azinda Ospedaliera Universitaria S. Giovanni Battista-Molinette di Torino \\
0132 & Outer West London Research Ethics Committee \\
0212 & Outer West London Research Ethics Committee \\
0204,0208 & CEIC Hospital Universitario I2 de Octubre \\
0136 & De Videnskabsetiske Komitéer for Region Hovedstaden Regionsgården \\
0205 & CMO Regio Arnhem Nijmegen \\
\hline
\end{tabular}

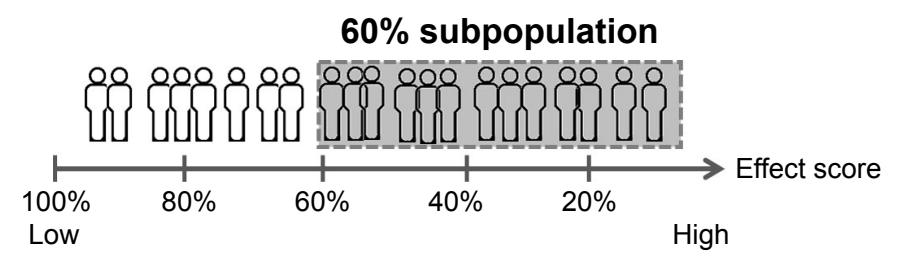

Figure SI Illustration of subpopulation identification based on effect score. ${ }^{a}$

Notes: apatients were ranked by their individual effect scores from highest to lowest and were sequentially grouped together as potential subpopulations by $10 \%$ increments, starting from the top $20 \%$ of patients with the highest individual effect score, until all patients (100\%) were included.

\section{Publish your work in this journal}

Therapeutics and Clinical Risk Management is an international, peerreviewed journal of clinical therapeutics and risk management, focusing on concise rapid reporting of clinical studies in all therapeutic areas, outcomes, safety, and programs for the effective, safe, and sustained use of medicines. This journal is indexed on PubMed Central, CAS,
EMBase, Scopus and the Elsevier Bibliographic databases. The manuscript management system is completely online and includes a very quick and fair peer-review system, which is all easy to use. Visit http://www.dovepress.com/testimonials.php to read real quotes from published authors.

Submit your manuscript here: http://www.dovepress.com/therapeutics-and-clinical-risk-management-journal 